Copyright (C1996, American Institute of Aeronautics and Astronautics, Inc.

AIAA Meeting Papers on Disc, 1996, pp. 212-223

A9634735, AIAA Paper 96-3596

\title{
Improved matched asymptotic solutions for three-dimensional atmospheric skip trajectories
}

\author{
Nguyen X. Vinh \\ Michigan Univ., Ann Arbor \\ Zeal-Sain Kuo \\ Chung Cheng Inst. of Technology, Taoyuan, Taiwan
}

\begin{abstract}
AIAA/AAS Astrodynamics Conference, San Diego, CA, July 29-31, 1996, Collection of Technical Papers (A96-34712 09-12), Reston, VA, American Institute of Aeronautics and Astronautics, 1996, p. 212-223
\end{abstract}

\begin{abstract}
An improved technique for matching the asymptotic solutions of nonlinear differential equations is presented and successfully applied to the 3D atmospheric skip trajectories. The classical method of matched asymptotic expansions is generally applied to two-point-boundary value problems. In the proposed technique, the second-order solutions are obtained by first generating a set of equations for the small perturbations which are the discrepancies between the uniformly valid first-order solutions and the exact solutions; the equations of the small perturbations are integrated separately near the outer and inner boundaries to obtain the perturbed outer and inner expansion solutions, respectively, for a second-order matching. In this technique, end-point boundaries are artificially extended to strengthen the physical assumptions on the outer and inner expansions for the matching, while in the evaluation of the constants of integration in the uniformly valid first-order solutions, the prescribed end-points are effectively enforced. Compared to solutions obtained by numerical integration over a wide range of entry conditions, the second-order solutions obtained by this improved technique are very accurate. (Author)
\end{abstract}




\title{
IMPROVED MATCHED ASYMPTOTIC SOLUTIONS FOR THREE-DIMENSIONAL ATMOSPHERIC SKIP TRAJECTORIES
}

\author{
Nguyen $X$. Vinh* \\ The University of Michigan \\ Ann Arbor, MI 48109-2118, U.S.A. \\ Zeal-Sain $\mathrm{Kuo}^{\dagger}$ \\ Chung Cheng Institute of Technology \\ Tao-Yuan, Taiwan, 33509, R.O.C.
}

\begin{abstract}
An improved technique for matching the asymptotic solutions of non-linear differential equations is presented and successfully applied to the three-dimensional atmospheric skip trajectories. The classical method of matched asymptotic expansions (MAE) is generally applied to two-point-boundary value problems. When we apply the MAE method to initial value problems, due to error propagation, the resulting accuracy usually depends on the physical problems. In the proposed technique, the second-order solutions are obtained by first generating a set of equations for the small perturbations which are the discrepancies between the uniformly valid first-order solutions and the exact solutions. Then, the equations of the small perturbations are integrated separately near the outer and inner boundaries to obtain the perturbed outer and inner expansion solutions, respectively, for a secondordcr matching. In addition, in this improved technique the end-point boundaries are artificially extended or constructed to strengthen the physical assumptions on the outer and inner expansions for the matching while in the evaluation of the constants of integration in the uniformly valid first-order solutions, the prescribed end-points are effectively enforced.

In this paper, to show the applicability of the improved technique, we first apply it to the rectilinear restricted three-body problem. We then consider the threedimensional skip trajectory. Compared to the solutions obtained by numerical integration over a wide range of entry conditions, the second-order solutions obtained by this improved technique are very accurate. The trajectory elements at the lowest altitude and at exit as well as their accuracy are evaluated.
\end{abstract}

Introduction

With the advent of manned space explorations and the establishment of permanent space stations, the safe

\footnotetext{
* Professor, Department of Aerospace Engineering. Member AIAA. $\uparrow$ Associate Professor, Dept. of Mechanical Enginecring. Copyright $\odot 1996$ by the American Institute of Aeronautics and Astronautics, Inc. All rights reserved.
}

recovery of an orbiting aerospace vehicle, or its orbital maneuver with minimum fuel consumption has been one of the most challenging technologies in space flight dynamics. During the atmospheric passage, there is a tremendous change in speed, kinetic energy, dynamic pressure and heating rate. It is then of interest to have explicit analytical solutions for the variations of the elements of the threc-dimensional entry trajectory, since the heading change due to three-dimensional motion has promising applications in aeroassisted orbital transfer. A powerful method for analyzing dynamic systems governed by equations with the dominant forces varying widely betwecn the two end-points is the method of matched asymptotic expansions (MAE). This technique, initiated by aerodynamicists ${ }^{1,2}$, has been successfully applied to problems in astrodynamics 3,4 . By using this method, some analytical solutions for atmospheric re-entry problems have been obtained, but they are restricted to the first-order solutions ${ }^{5,6,7}$. In this paper, we propose an improved technique to go beyond the first-order solutions reported previously. In this improved technique, the perturbation equations are generated by considering the small discrepancies between the exact solutions and the uniformly valid first-order solutions. Then, the equations for the small perturbations are intcgratcd scparatcly near the outer and inner boundaries to obtain the perturbed outer and inner expansion solutions, respectively, for a second-order matching.

To illustrate the applicability of the improved method of matched asymptotic expansions (iMAE), the proposed technique was first applied to the rectilinear restricted three-body problem. Then, by using it to analyze the three-dimensional atmospheric re-entry problem, we obtain, besides the usual solutions for the altitude, speed and flight path angle variables, the secondorder solutions for the heading, latitude and longitude in explicit form with excellent accuracy. The explicit second-order solutions are compared with the pure numerical solutions and the errors incurred are assessed to show the region of validity for the application of the technique. 
Rectilinear Restricted Three-Body Problem

We first consider an example in the restricted threebody problem. The motion of a space vehicle of mass $m$ in the gravitational field of two fixed mass-centers, $M_{1}$ and $M_{2}$ is shown in Fig. 1.

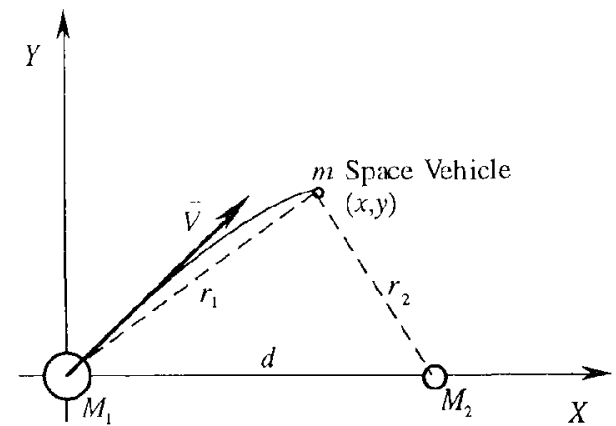

Fig. 1 Geometry of the restricted three-body problem

The mass of the vehicle is much smaller than the other two bodies, and the mass $M_{1}$ is much larger than the mass $M_{2}$. The equations of motion with respect to the rectangular Cartesian coordinate system in dimensionless form are

$$
\begin{aligned}
& \frac{d^{2} x}{d t^{2}}=-(1-\mu) \frac{x}{r_{1}^{3}}-\mu \frac{x-1}{r_{2}^{3}} \\
& \frac{d^{2} y}{d t^{2}}=-(1-\mu) \frac{y}{r_{1}^{3}}-\mu \frac{y}{r_{2}^{3}}
\end{aligned}
$$

where

$$
r_{1}^{2}=x^{2}+y^{2}, \quad r_{2}^{2}=(x-1)^{2}+y^{2}
$$

and

$$
\mu=\frac{M_{2}}{M_{1}+M_{2}}
$$

The position vector $(x, y)$ was normalized by the distance $d$ between the two mass centers. The time was made dimensionless using the factor

$$
\left[\frac{d^{3}}{G\left(M_{1}+M_{2}\right)}\right]^{1 / 2}
$$

where $G$ is the universal gravitational constant. This problem has been studied by Lagerstrom and Kevorkian ${ }^{3}$ for the initial conditions

$$
x=0, y=0, \frac{d y}{d x}=-\mu c \text { and } h=-\rho^{2} \text { at } t=0
$$

where $h$ is the total energy of the space vehicle and $\rho \neq 1$. When the infinitesimal mass is close to the smaller of the two masses, the proposed stretching transformation is

$$
\bar{x}=\frac{1-x}{\mu^{\alpha}}, \bar{y}=\frac{y}{\mu^{\alpha}}, \bar{t}=\frac{t-t_{g}}{\mu^{\beta}}
$$

where $\alpha, \beta>0$, and $t_{e}$ is the time elapsed to reach $x=1$. It can be shown by an analysis of the order of magnitude of the various terms in the equations that $\alpha=1$ and $\beta=$ 1. With the new variables, $\bar{x}=(1-x) / \mu$ and $\bar{y}=y / \mu$, the original equations can be rewritten in terms of the inner variables as

$$
\begin{aligned}
& \mu \frac{d^{2} \bar{x}}{d t^{2}}=\frac{(1-\mu)(1-\mu \bar{x})}{\left[(1-\mu \bar{x})^{2}+(\mu \bar{y})^{2}\right]^{3 / 2}}-\frac{\mu^{2} \bar{x}}{\left[(\mu \bar{x})^{2}+(\mu \bar{y})^{2}\right]^{3 / 2}} \\
& \mu \frac{d^{2} \bar{y}}{d t^{2}}=\frac{-\mu(1-\mu) \bar{y}}{\left[(1-\mu \bar{x})^{2}+(\mu \bar{y})^{2}\right]^{3 / 2}}-\frac{\mu^{2} \bar{y}}{\left[(\mu \bar{x})^{2}+(\mu \bar{y})^{2}\right]^{3 / 2}}
\end{aligned}
$$

To illustrate the improved method, we simply consider the onc-dimensional case. If we set $y=0$ and integrate (1) with the total energy equals to zero, we have

$$
\frac{1}{2}\left(\frac{d x}{d t}\right)^{2}=\frac{1-\mu}{x}+\frac{\mu}{1-x}, \quad t(0)=0
$$

or in the new form

$$
\begin{aligned}
\sqrt{2} \frac{d t}{d x} & =\sqrt{\frac{x(1-x)}{(1-x)+\mu(2 x-1)}} \\
& =\sqrt{x}-\frac{\mu}{2} \sqrt{x}\left(\frac{2 x-1}{1-x}\right)+\mu^{2} \frac{3}{8} \sqrt{x}\left(\frac{2 x-1}{1-x}\right)^{2}+\cdots
\end{aligned}
$$

We anticipate the outer expansion in the form

$$
t^{o}=t_{0}+\mu t_{1}+\mu^{2} t_{2}+\cdots
$$

By using the outer limit, $\mu \rightarrow 0$, with $x$ fixed, the firstterm of the outer expansion is

$$
\sqrt{2} t_{0}=\frac{2}{3} x^{3 / 2}+C_{0}
$$

where $C_{0}$ is the constant of integration.

For the inner solution, that is the solution near $x=$ 1 , if we let $\bar{y}=0$ in the equation (8) and integrate the resulting equation, we obtain

$$
\frac{1}{2} \mu^{2}\left(\frac{d \bar{x}}{d t}\right)^{2}=\frac{1-\mu}{1-\mu \bar{x}}+\frac{1}{\bar{x}}
$$

or in the new form 


$$
\sqrt{2} \frac{d t}{d \bar{x}}=\mu \sqrt{\frac{(1-\mu \bar{x}) \bar{x}}{\bar{x}+1-2 \mu \bar{x}}}
$$

It is clear that the first term of the inner solution is the constant of integration because the $O(1)$ term in the righthand side of the equation (15) is zero. Therefore, we have

$$
\sqrt{2} \bar{t}_{0}=\bar{C}_{0}
$$

where $\bar{t}_{0}$ is the first term of the inner expansion

$$
t^{i}=\bar{t}_{0}(\bar{x})+\mu \bar{t}_{1}(\bar{x})+\mu^{2} \bar{t}_{2}(\bar{x})+\cdots
$$

Since the first term of the inner expansion is a constant, the first-order composite solution is identical to the outer solution due to a cancellation of the constant $\bar{C}_{0}$ which is the common term in the matching process. Thus, we have

$$
\sqrt{2} t_{c}=\frac{2}{3} x^{3 / 2}+C_{0}
$$

With the initial condition $t(0)=0$, the constant $C_{0}$ is equal to zero.

We obtain the second-order composite solution by generating the equation for the perturbation between the first-order composite solution and the exact solution, then integrate it by using the MAE method again. Let the perturbation be

$$
q=\sqrt{2} t-\sqrt{2} t_{c}
$$

By substituting into the original equation (11), we have

$$
\begin{aligned}
\frac{d q}{d x} & =\sqrt{\frac{x(1-x)}{(1-x)+\mu(2 x-1)}}-\sqrt{x} \\
& =-\frac{\mu}{2} \sqrt{x}\left(\frac{2 x-1}{1-x}\right)+\mu^{2} \frac{3}{8} \sqrt{x}\left(\frac{2 x-1}{1-x}\right)^{2}+\cdots
\end{aligned}
$$

with the initial condition

$$
q(0)=0
$$

It can be easily shown that the outer expansion is

$$
\begin{aligned}
q^{o} & =\mu\left[\frac{2}{3} x^{3 / 2}+\sqrt{x}-\frac{1}{2} \ln \left(\frac{1+\sqrt{x}}{1-\sqrt{x}}\right)+C_{1}\right]+O\left(\mu^{2}\right) \\
& =\mu\left[\frac{2}{3} x^{3 / 2}+\sqrt{x}-\ln (1+\sqrt{x})+\frac{1}{2} \ln \mu\right. \\
& \left.+\frac{1}{2} \ln \frac{1-x}{\mu}+C_{1}\right]+O\left(\mu^{2}\right)
\end{aligned}
$$

where $C_{1}$, the constant of integration, will be determined after matching.

To describe the inner solution near $x=1$, we write (20) in terms of inner variable

$$
\begin{aligned}
\frac{d q}{d \bar{x}} & =-\mu\left[\sqrt{\frac{(1-\mu \bar{x}) \bar{x}}{\bar{x}+1-2 \mu \bar{x}}}-\sqrt{1-\mu \bar{x}}\right] \\
& =-\mu\left[\sqrt{\frac{\bar{x}}{1+(1-2 \mu) \bar{x}}}-1\right]\left(1-\frac{\mu}{2} \bar{x}+\mu^{2} \frac{1}{8} \bar{x}^{2}+\cdots\right)
\end{aligned}
$$

Again, since the right-hand side is of $O(\mu)$, we seek an inner expansion of the perturbation in the form

$$
q^{i}=\mu\left[\bar{q}_{0}(\bar{x})+\mu \bar{q}_{1}(\bar{x})+\mu^{2} \bar{q}_{2}(\bar{x})+\cdots\right]
$$

Hence, upon substituting into equation (23) and integrating, the inner expansion is

$$
\begin{aligned}
q^{i} & =\mu\left[\bar{x}-\frac{\sqrt{(1-2 \mu) \bar{x}^{2}+\bar{x}}}{1-2 \mu}\right. \\
& \left.+\frac{\sinh ^{-1} \sqrt{(1-2 \mu) \bar{x}}}{(1-2 \mu)^{3 / 2}}+\bar{C}_{1}\right]+O\left(\mu^{2}\right)
\end{aligned}
$$

where $\bar{C}_{1}$ is a constant to be determined from matching.

To perform the matching, we use a generalized form of the matching principle:

The inner expansion of (the outer expansion) equals the outer expansion of (the inner expansion)

Thus, we let

$\left(q^{o}\right)^{i} \equiv$ the inner expansion of (the outer expansion)

$$
\begin{aligned}
& =\mu\left[\left(\frac{5}{3}-\frac{3}{2} \mu \bar{x}+\cdots\right)-\ln (1+\sqrt{1-\mu \bar{x}})\right. \\
& \left.+\frac{1}{2} \ln \mu+\frac{1}{2} \ln \bar{x}+C_{1}\right]+O\left(\mu^{2}\right)
\end{aligned}
$$

and

$\left(q^{i}\right)^{0} \equiv$ the outer expansion of (the inner expansion)

$$
\begin{aligned}
& =\mu\left[-(1-x)-\left(\frac{1}{2(1-2 \mu)}+\cdots\right)\right. \\
& \left.+\frac{\frac{1}{2} \ln \left(\frac{1-x}{\mu}\right)+\ln 2+\cdots}{(1-2 \mu)^{3 / 2}}+\bar{C}_{1}\right]+O\left(\mu^{2}\right)
\end{aligned}
$$


By equating (26) and (27) according to the matching principle and taking the limit, $\mu \rightarrow 0$, we have

$$
\bar{C}_{1}=\frac{13}{6}-2 \ln 2+\frac{1}{2} \ln \mu+C_{1}
$$

The common part $q_{o o}$ is

$$
\begin{aligned}
& q_{o o}=\left(q^{i}\right)^{o}=\mu\left[-(1-x)-\frac{1}{2}\right. \\
& \left.+\frac{1}{2} \ln \left(\frac{1-x}{\mu}\right)+\ln 2+\bar{C}_{1}\right]+O\left(\mu^{2}\right)
\end{aligned}
$$

A composite solution for the perturbation $q$ can be obtained as

$$
\begin{aligned}
q^{c} & =q^{o}+q^{i}-\left(q^{i}\right)^{o} \\
& =\mu\left[\frac{2}{3} x^{3 / 2}+\sqrt{x}-\ln (1+\sqrt{x})\right. \\
& +\bar{x}-\frac{\sqrt{(1-2 \mu) \bar{x}^{2}+\bar{x}}}{1-2 \mu}+\frac{\sinh ^{-1} \sqrt{(1-2 \mu) \bar{x}}}{(1-2 \mu)^{3 / 2}} \\
& \left.+(1-x)+\frac{1}{2}-\ln 2+\frac{1}{2} \ln \mu+C_{1}\right]+O\left(\mu^{2}\right)
\end{aligned}
$$

where $C_{1}$ is evaluated by using the initial condition $q^{c}(0)=0$. Thus, the improved second-order composite solution uniformly valid over $[0,1]$ can be formed according to

$$
\sqrt{2} t=\frac{2}{3} x^{3 / 2}+q
$$

Therefore, we obtain the second-order solution

$$
\begin{aligned}
\sqrt{2} t^{c}= & \frac{2}{3} x^{3 / 2}+q^{c} \\
& =\frac{2}{3} x^{3 / 2}+\mu\left[\frac{2}{3} x^{3 / 2}+\sqrt{x}-\ln (1+\sqrt{x})\right. \\
& +\bar{x}-\frac{\sqrt{(1-2 \mu) \bar{x}^{2}+\bar{x}}}{1-2 \mu}+\frac{\sinh ^{-1} \sqrt{(1-2 \mu) \bar{x}}}{(1-2 \mu)^{3 / 2}} \\
& \left.+(1-x)+\frac{1}{2}-\ln 2+\frac{1}{2} \ln \mu+C_{1}\right]+O\left(\mu^{2}\right)
\end{aligned}
$$

By using classical MAE, Nayfeh ${ }^{8}$ obtained the following solution denoted by $t_{N}^{c}$

$$
\begin{aligned}
\sqrt{2} t_{N}^{c} & =\frac{2}{3} x^{3 / 2}+\mu\left[\frac{2}{3} x^{3 / 2}+\sqrt{x}-\ln (1+\sqrt{x})\right. \\
& +\bar{x}-\sqrt{\bar{x}(\bar{x}+1)}+\sinh ^{-1} \sqrt{\bar{x}} \\
& \left.+\frac{1}{2}-\ln 2+\frac{1}{2} \ln \mu\right]+O\left(\mu^{2}\right)
\end{aligned}
$$

The improved second-order composite solution (32) denoted by iMAE is graphed in Figs. 2 and 3 for comparison with the numerical solution and Nayfeh's solution (33). It is seen that the improved method has better accuracy than the classical method.

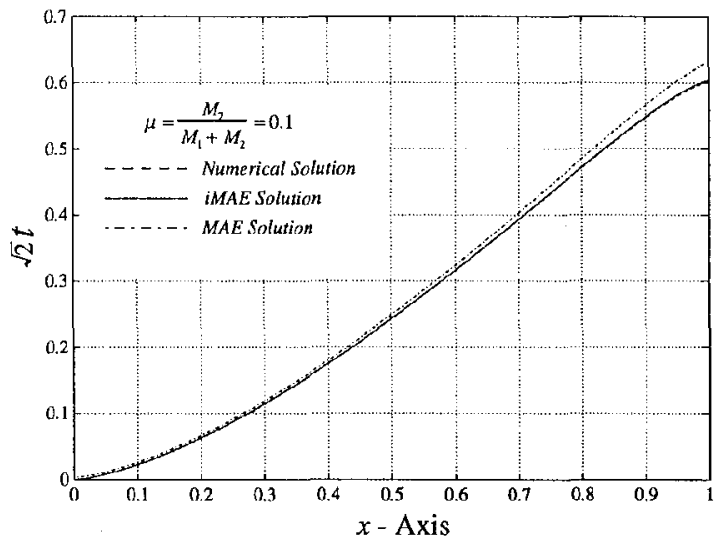

Fig. 2 The dimensionless time as function of the distance $x$ in the one-dimensional restricted three-body problem when the total energy is zero, and $\mu=0.1$

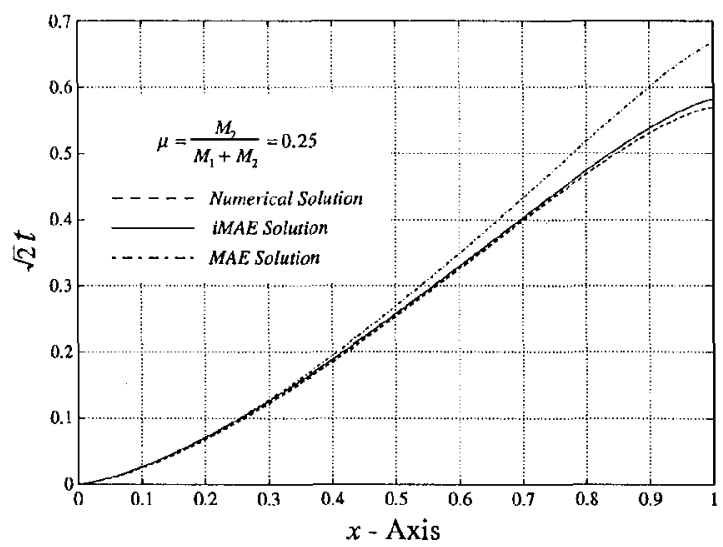

Fig. 3 The dimensionless time as function of the distance $x$ in the one-dimensional restricted three-body problem when the total energy is zero, and $\mu=0.25$

\section{Three-Dimensional Atmospheric Skip Trajectory}

\section{Fundamental Equations of Motion}

We now apply the technique to the calculation of the three-dimensional flight trajectory of a space vehicle entering a non-rotating planetary atmosphere. Using the standard notation as shown in Fig. 4, we have the governing equations 


$$
\begin{aligned}
\frac{d r}{d t} & =V \sin \gamma \\
\frac{d V}{d t} & =-\frac{\rho A C_{D} V^{2}}{2 m}-g \sin \gamma \\
V \frac{d \gamma}{d t} & =\frac{\rho A C_{L} V^{2}}{2 m} \cos \sigma-\left(g-\frac{V^{2}}{r}\right) \cos \gamma \\
V \frac{d \psi}{d t} & =\frac{\rho A C_{L} V^{2}}{2 m \cos \gamma} \sin \sigma-\frac{V^{2}}{r} \cos \gamma \cos \psi \tan \phi \\
\frac{d \phi}{d t} & =\frac{V \cos \gamma \sin \psi}{r} \\
\frac{d \theta}{d t} & =\frac{V \cos \gamma \cos \psi}{r \cos \phi}
\end{aligned}
$$

For a Newtonian inverse-square gravitational field we have

$$
\frac{g}{g_{s}}=\frac{r_{s}^{2}}{r^{2}}
$$

where subscript $s$ denotes the reference sea level.

We define the dimensionless variables

$$
u=\frac{V^{2}}{g_{s} r_{s}}, \quad h=\frac{r-r_{s}}{r_{s}}
$$

for the speed and the altitude.

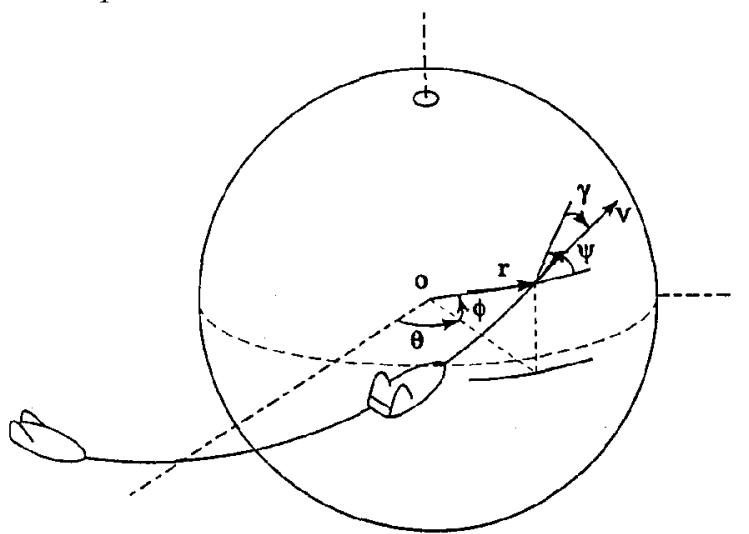

Fig. 4. Coordinate system with trajectory variables

Furthermore, we use a strictly exponential atmospherc of the form

$$
\rho=\rho_{s} e^{-h / \varepsilon}, \quad \varepsilon=\frac{1}{\beta r_{s}}
$$

where $\varepsilon$ is a small dimensionless parameter. form

The lift and drag coefficients are assumed to be of the

and

$$
C_{L}=\lambda C_{L}^{*}
$$

$$
C_{D}=\frac{C_{D}^{*}}{2}\left(1+\lambda^{2}\right)
$$

where $C_{L}^{*}$ and $C_{D}^{*}$ are respectively the lift coefficient and the drag coefficient at maximum lift-to-drag ratio and $\lambda$ is the normalized lift coefficient. Then, by using the dimensionless altitude $h$ as the independent variable to replace the time variable, we have the dimensionless equations of motion

$$
\begin{aligned}
& \frac{d u}{d h}=-\frac{2}{(1+h)^{2}}-\frac{B\left(1+\lambda^{2}\right) u e^{-h / \varepsilon}}{\varepsilon E^{*} \sin \gamma} \\
& \frac{d \gamma}{d h}=\left[\frac{1}{(1+h)}-\frac{1}{u(1+h)^{2}}\right] \frac{1}{\tan \gamma}+\frac{B \lambda \cos \sigma e^{-h / \varepsilon}}{\varepsilon \sin \gamma} \\
& \frac{d \psi}{d h}=-\frac{\cos \psi \tan \phi}{(1+h) \tan \gamma}+\frac{B \lambda \sin \sigma e^{-h / \varepsilon}}{\varepsilon \sin \gamma \cos \gamma} \\
& \frac{d \phi}{d h}=\frac{\sin \psi}{(1+h) \tan \gamma} \\
& \frac{d \theta}{d h}=\frac{\cos \psi}{(1+h) \tan \gamma \cos \phi}
\end{aligned}
$$

In the equations, we have a small dimensionless parameter $\varepsilon$ representing the planetary atmosphere characteristic. For the Earth's atmosphere we have the practical value of $\varepsilon=1 / 900$. Furthermore, the maximum lift-to-drag ratio $E^{*}=C_{L}^{*} / C_{D}^{*}$ represents the performance characteristic of the lifting vehicle, while other physical parameters are lumped together in the coefficient $B$

defined as

$$
B=\frac{\rho_{s} A C_{L}^{*}}{2 m \beta}
$$

The flight program is specified by the normalized lift coefficient $\lambda$ and the bank angle $\sigma$.

The system (40) constitutes the most appropriate dimensionless system of equations for analyzing entry into a planetary atmosphere, with lift and bank modulation. To generate the three-dimensional entry trajectory, it suffices to select a lift control $\lambda(h)$ and a bank anglc $\sigma(h)$ as functions of the altitude and integrate the equations from the initial condition $\left[u_{i}, \gamma_{i}, \psi_{i}, \phi_{i}, \theta_{i}\right]$ at entry altitude $h_{i}$. In subsequent computation we shall use a constant angle-of-attack, that is a constant $\lambda$ and constant bank angle $\sigma$ for the flight.

Integration by Matched Asymptotic Expansions By using the matched asymptotic expansions method, we obtain separately the outer solutions at high altitude by retaining in the equations only the dominant gravitational and centrifugal force and the inner solutions at low altitude by keeping only the strong aerodynamic force. By using appropriate matching, a composite solution uniformly valid over the whole entry trajectory is then obtained. 


\section{Outer Expansions (Keplerian Region)}

The outer expansions describe the behavior of the motion in the region near the vacuum. At high altitude in the limiting case, $e^{-h / \varepsilon} \rightarrow 0$, the aerodynamic force nearly disappears when compared with the combination of the gravitational and centrifugal forces. Therefore, we obtain the outer expansions by repeated application of the outer limit, $\varepsilon \rightarrow 0$ with the altitude variable $h$ and other variables held fixed. We consider the state vector $x=[u, \gamma, \psi, \phi, \theta]$ and assume the following expansions

$$
x^{\circ}=x_{o}(h)+\varepsilon x_{1}(h)+\varepsilon^{2} x_{2}(h)+\ldots
$$

By substituting into the system (40) and taking the outer limit, the first-order equations for the outer expansions are

$$
\begin{aligned}
\frac{d u_{o}}{d h} & =-\frac{2}{(1+h)^{2}} \\
\frac{d \gamma_{o}}{d h} & =\left\{\frac{1}{(1+h)}-\frac{1}{u_{o}(1+h)^{2}}\right\} \frac{1}{\tan \gamma_{o}} \\
\frac{d \psi_{o}}{d h} & =-\frac{\cos \psi_{o} \tan \phi_{o}}{(1+h) \tan \gamma_{o}} \\
\frac{d \phi_{o}}{d h} & =\frac{\sin \psi_{o}}{(1+h) \tan \gamma_{o}} \\
\frac{d \theta_{o}}{d h} & =\frac{\cos \psi_{o}}{(1+h) \tan \gamma_{o} \cos \phi_{o}}
\end{aligned}
$$

These are the equations for three-dimensional Keplerian motion in the vacuum. Upon integrating, the outer solutions are

$$
\begin{aligned}
u_{o} & =\frac{2}{(1+h)}+C_{1} \\
\cos \gamma_{o} & =\frac{C_{2}}{\sqrt{C_{1}(1+h)^{2}+2(1+h)}} \\
\cos \psi_{o} & =\frac{C_{3}}{\cos \phi_{o}} \\
\sin \phi_{o} & =\sqrt{1-C_{3}^{2}} \sin \left\{\cos ^{-1}\left(\frac{1-\frac{C_{2}^{2}}{1+h}}{\sqrt{1+C_{1} C_{2}^{2}}}\right)+C_{4}\right\} \\
\cos \left(\theta_{o}-C_{5}\right) & \left.=\frac{\cos \left\{\cos ^{-1}\left(\frac{1-\frac{C_{2}^{2}}{1+h}}{\sqrt{1+C_{1} C_{2}^{2}}}\right)+C_{4}\right\}}{\cos \phi_{o}}\right\}
\end{aligned}
$$

where $C_{n}, n=1,2, \cdots, 5$ are the constants of integration to be determined after matching.

\section{Inner Expansions (Aerodynamic Predominant Region)}

Near the surfacc of the planet, the aerodynamic force is dominant. The inner expansions are obtained by repeated application of the inner limit which is defined as the limit when $\varepsilon \rightarrow 0$ with the new altitude variable $\bar{h}=h / \varepsilon$ and the other dimensionless variables held fixed. We assume the following expansion

$$
x^{i}=\bar{x}_{o}(\bar{h})+\varepsilon \bar{x}_{1}(\bar{h})+\varepsilon^{2} \bar{x}_{2}(\bar{h})+\ldots
$$

By substituting into the system (40) and taking the inner limit, the first-order equations for the inner expansions are

$$
\begin{aligned}
& \frac{d \bar{u}_{o}}{d \bar{h}}=-\frac{B\left(1+\lambda^{2}\right) \bar{u}_{o} e^{-\bar{h}}}{E^{*} \sin \bar{\gamma}_{o}} \\
& \frac{d \bar{\gamma}_{o}}{d \bar{h}}=\frac{B \lambda \cos \sigma e^{-\bar{h}}}{\sin \bar{\gamma}_{o}} \\
& \frac{d \bar{\psi}_{o}}{d \bar{h}}=\frac{B \lambda \sin \sigma e^{-\bar{h}}}{\sin \bar{\gamma}_{o} \cos \bar{\gamma}_{o}} \\
& \frac{d \bar{\phi}_{o}}{d \bar{h}^{\prime}}=0 \\
& \frac{d \bar{\theta}_{o}}{d \bar{h}}=0
\end{aligned}
$$

This system can also be integrated to yield the inner solutions

$$
\begin{aligned}
\bar{u}_{o} & =\bar{C}_{1} e^{-\frac{\left(1+\lambda^{2}\right)}{\lambda E^{\prime} \cos \sigma} \bar{\gamma}_{o}} \\
\cos \bar{\gamma}_{o} & =B \lambda \cos \sigma e^{-h / \varepsilon}+\bar{C}_{2} \\
\bar{\psi}_{o} & =\tan \sigma \ln \left(\sec \bar{\gamma}_{o}+\tan \bar{\gamma}_{o}\right)+\bar{C}_{3} \\
\bar{\phi}_{o} & =\bar{C}_{4} \\
\bar{\theta}_{o} & =\bar{C}_{5}
\end{aligned}
$$

where $\bar{C}_{n}, n=1,2, \cdots, 5$ are the corresponding constants of integration.

\section{First-Order Composite Solutions}

To have the solutions uniformly valid over both the outer and the inner regions, we construct the composite solutions by taking the sum of the outer and inner solutions and subtracting the parts they have in common.

$$
x_{c}=x_{o}+\bar{x}_{o}-x_{o o}
$$

where $x_{o o}$ is the common limit. To avoid state variables becoming imaginary before the vehicle reaches the lowest altitude, we require that at a certain altitude $h_{b}$ the 
composite solution $\gamma_{c}$ and the inner solution $\bar{\gamma}_{o}$ reach zero simultaneously, that is,

$$
\bar{\gamma}_{o}\left(h_{b} / \varepsilon\right)=\gamma_{c}\left(h=h_{b}\right)=0
$$

Hence, for matching, we choose a modified common limit such that equation (49) is satisfied

$$
x_{o o}=\lim _{h \rightarrow h_{b}} x_{o}(h)=\lim _{\bar{h} \rightarrow \infty} \bar{x}_{o}(\bar{h})
$$

i. e.,

$$
\begin{aligned}
& u_{o o}=\frac{2}{1+h_{b}}+C_{1}=\bar{C}_{1} e^{-\frac{\left(1+\lambda^{2}\right)}{\lambda E^{*} \cos \sigma} \gamma_{o o}} \\
& \cos \gamma_{o o}=\frac{C_{2}}{\sqrt{C_{1}\left(1+h_{b}\right)^{2}+2\left(1+h_{b}\right)}}=\bar{C}_{2} \\
& \psi_{o o}=\cos ^{-1}\left(\frac{C_{3}}{\cos \phi_{o o}}\right) \\
& =\tan \sigma \ln \left[\sec \gamma_{o o}+\tan \gamma_{o o}\right]+\bar{C}_{3} \\
& \sin \phi_{o o}=\sqrt{1-C_{3}^{2}} \sin \alpha=\sin \bar{C}_{4} \\
& \cos \left(\theta_{o o}-C_{5}\right)=\frac{\cos \alpha}{\cos \phi_{o o}}=\cos \left(\bar{C}_{5}-C_{5}\right)
\end{aligned}
$$

where we have defined

$$
\alpha \equiv \cos ^{-1}\left(\frac{1-\frac{C_{2}^{2}}{1+h}}{\sqrt{1+C_{1} C_{2}^{2}}}\right)+C_{4}
$$

Then, according to equation (48), the first-order composite solutions are

$$
\begin{aligned}
u_{c}= & \frac{2}{1+h}+\bar{C}_{1} e^{-\frac{\left(1+\lambda^{2}\right)}{\lambda E^{*} \cos \sigma} \cos ^{-1}\left(B \lambda \cos \sigma e^{-h / \varepsilon}+\bar{C}_{2}\right)}-\frac{2}{1+h_{b}} \\
\cos \gamma_{c} & =\frac{C_{2}}{\sqrt{C_{1}(1+h)^{2}+2(1+h)}}+B \lambda \cos \sigma e^{-h / \varepsilon} \\
\psi_{c} & =\cos ^{-1}\left(\frac{C_{3}}{\cos \phi_{c}}\right)+\tan \sigma \ln \left(\sec \bar{\gamma}_{o}+\tan \bar{\gamma}_{o}\right)+\bar{C}_{3} \\
& -\cos ^{-1}\left(\frac{C_{3}}{\cos \phi_{o o}}\right)
\end{aligned}
$$

$\sin \phi_{c}=\sqrt{1-C_{3}^{2}} \sin \alpha$

$\cos \left(\theta_{c}-C_{5}\right)=\frac{\cos \alpha}{\cos \phi_{c}}$

From the second of the equations (52), at the lowest altitude we have

$$
1=B \lambda \cos \sigma e^{-h_{b} / \varepsilon}+\bar{C}_{2}
$$

Now, there are eleven unknowns, namcly the ten constants of integration and the lowest altitude $h_{b}$. If we set the initial conditions, $\left[u_{i}, \gamma_{i}, \psi_{i}, \phi_{i}, \theta_{i}\right]$ at $h_{i}$, in the composite solutions (52) to be identically satisfied, these 5 new equations, together with the equations (51) and (53) constitute a system of 11 equations for 11 unknowns. Since the first two variables $u$ and $\gamma$ are independent of the other variables $\psi, \phi$, and $\theta$, we can first solve for $C_{1}, C_{2}, \bar{C}_{1}, \bar{C}_{2}$ and $h_{b}$. Next, from the relevant equations, we solve for $C_{3}, C_{4}, \bar{C}_{3}$ and $\bar{C}_{4}$. Finally, the constants of integration $C_{5}$ and $\bar{C}_{5}$ for $\theta$ can be evaluated by satisfying the matching condition and the initial condition for $\theta$ at $h_{i}$. With the constants evaluated, the equations (52) are the first-order composite solutions in terms of the altitude variable $h$.

\section{Second-Order Solutions}

We construct the second-order solutions by considering the small discrepancies between the first-order composite solutions and the exact solutions. Let

$$
\begin{aligned}
u & =u_{c}+z=u_{o}+\bar{u}_{o}-u_{o o}+z \\
\cos \gamma & =\cos \gamma_{c}+q=\cos \gamma_{o}+\cos \bar{\gamma}_{o}-\cos \gamma_{o o}+q \\
\psi & =\psi_{c}+y=\psi_{o}+\bar{\psi}_{o}-\psi_{o o}+y \\
\phi & =\phi_{c}+f=\phi_{o}+\bar{\phi}_{o}-\phi_{o o}+f=\phi_{o}+f \\
\theta & =\theta_{c}+p=\theta_{o}+\bar{\theta}_{o}-\theta_{o o}+p=\theta_{o}+p
\end{aligned}
$$

Notice that because the inner solutions $\bar{\phi}_{o}$ and $\bar{\theta}_{o}$ are constants, they are canceled by their common limits $\phi_{o o}$ and $\theta_{o o}$, respectively. As a consequence, the classical first-order composite solutions for $\theta$ and $\phi$ are not sufficiently accurate since they are simply the Keplerian solutions. By substituting equations (54) into the dimensionless equations of motion (40) and using the equations of outer and inner expansions for simplification, we obtain the equations for the small perturbations

$$
\begin{aligned}
\frac{d z}{d h} & =-\frac{B\left(1+\lambda^{2}\right) u e^{-h / \varepsilon}}{\varepsilon E^{*} \sin \gamma}+\frac{B\left(1+\lambda^{2}\right) \bar{u}_{o} e^{-h / \varepsilon}}{\varepsilon E^{*} \sin \bar{\gamma}_{o}} \\
\frac{d q}{d h} & =-\left\{\frac{1}{(1+h)}-\frac{1}{u(1+h)^{2}}\right\} \cos \gamma \\
& +\left\{\frac{1}{(1+h)}-\frac{1}{u_{o}(1+h)^{2}}\right\} \cos \gamma_{o}
\end{aligned}
$$




$$
\begin{aligned}
\frac{d y}{d h}= & -\frac{1}{(1+h)}\left(\frac{\cos \psi \tan \phi}{\tan \gamma}-\frac{\cos \psi_{o} \tan \phi_{o}}{\tan \gamma_{o}}\right) \\
& +\left(\frac{B \lambda \sin \sigma e^{-h / \epsilon}}{\varepsilon \sin \gamma \cos \gamma}-\frac{B \lambda \sin \sigma e^{-h / \varepsilon}}{\varepsilon \sin \bar{\gamma}_{o} \cos \bar{\gamma}_{o}}\right) \\
\frac{d f}{d h} & =\frac{1}{(1+h)}\left(\frac{\sin \psi}{\tan \gamma}-\frac{\sin \psi_{o}}{\tan \gamma_{o}}\right) \\
\frac{d p}{d h} & =\frac{1}{(1+h)}\left(\frac{\cos \psi}{\tan \gamma \cos \phi}-\frac{\cos \psi_{o}}{\tan \gamma_{o} \cos \phi_{o}}\right)
\end{aligned}
$$

The initial conditions for the perturbations are trivially

$$
z\left(h_{i}\right)=0, q\left(h_{i}\right)=0, y\left(h_{i}\right)=0, f\left(h_{i}\right)=0, p\left(h_{i}\right)=0
$$

We integrate the equations for the perturbations separately first in the outer region and then in the inner region.

\section{Second-Order Solutions for $u$ and $\gamma$}

Since the equation for $u$ and $\gamma$ are decouplcd from the system, we can integrate the first two equations by assuming the outer expansions for the perturbations

$$
\begin{aligned}
& z^{o}=z_{o}(h)+\varepsilon z_{1}(h)+\varepsilon^{2} z_{2}(h)+\cdots \\
& q^{o}=q_{o}(h)+\varepsilon q_{1}(h)+\varepsilon^{2} q_{2}(h)+\cdots
\end{aligned}
$$

By taking the outer limit, $\varepsilon \rightarrow 0$, while keeping other variables fixed, we obtain the outer solutions for the perturbation $z$

$$
\frac{d z_{o}}{d h}=0 \Rightarrow z_{o}=C_{6}
$$

It can be shown that $C_{6}$ is zero. Then, upon using this solution and integrating, the outer solutions for the perturbation $q$ is

$$
q_{o}=\frac{C_{7}}{\sqrt{C_{1}(1+h)^{2}+2(1+h)}}
$$

where $C_{7}$ is the constant of integration.

For the inner solution $z$ since $\sin \gamma$ will tend to $\sin \bar{\gamma}_{o}$ and is very small, by inspecting the first of the perturbation equations, it is seen that $u$ is very close to $\bar{u}_{o}$. This makes the evaluation of $z$ very sensitive to errors. Since the first-order composite solution for $u$ is sufficiently accurate, we concentrate on improving the solution for the perturbation of the flight path angle in the inner region. First, we anticipate the inner expansions for the perturbations in the form

$$
\begin{aligned}
& z^{i}=\bar{z}_{o}(\bar{h})+\varepsilon \bar{z}_{1}(\bar{h})+\varepsilon^{2} \bar{z}_{2}(\bar{h})+\cdots \\
& q^{i}=\varepsilon\left[\bar{q}_{o}(\bar{h})+\varepsilon \bar{q}_{1}(\bar{h})+\varepsilon^{2} \bar{q}_{2}(\bar{h})+\cdots\right]
\end{aligned}
$$

Next, we use the following limiting and approximate conditions

$$
\begin{aligned}
& u \rightarrow \bar{u}_{o}+z \approx \bar{u}_{o}, \quad z \ll 1 \\
& \cos \gamma \rightarrow \cos \bar{\gamma}_{o}+q \approx \cos \bar{\gamma}_{o}, \quad q \ll 1 \\
& 1+h \rightarrow 1+h_{b}
\end{aligned}
$$

By taking the inner limit, while keeping other variables fixed, we obtain the equation for $\bar{q}_{0}$

$$
\begin{aligned}
\frac{d \bar{q}_{o}}{d \bar{h}} & =-\left\{\frac{1}{(1+\varepsilon \bar{h})}-\frac{1}{\bar{u}_{o}\left(1+h_{b}\right)^{2}}\right\} \cos \bar{\gamma}_{o} \\
& +\left\{\frac{1}{(1+\varepsilon \bar{h})}-\frac{1}{u_{o}(1+\varepsilon \bar{h})^{2}}\right\} \cos \gamma_{o}
\end{aligned}
$$

Notice here in (62) we keep the integrable terms in exact form to increase accuracy and at the same time make the final solution form simple. Therefore, only in the second term that $\varepsilon \bar{h}$ is replaced by $h_{b}$. Upon integrating it, we have the inner solution for the perturbation $q$ in the form

$$
\bar{q}_{o}=f(\bar{h})+\bar{C}_{7}
$$

The expression for $f(\bar{h})$ is much involved so that we simply discuss its application. We use this solution (63) and the outer solution $q_{o}$ in Eq. (59) to construct the composite solution $q_{c}(h)$ satisfying identically the initial condition $q\left(h_{i}\right)=0$. Then, the second-order solution for $\gamma$ is

$$
\begin{aligned}
\cos \gamma^{c} & =\cos \gamma_{c}+q_{c} \\
& =\frac{C_{2}}{\sqrt{C_{1}(1+h)^{2}+2(1+h)}}+B \lambda \cos \sigma e^{-h / \varepsilon}+q_{c}(h)
\end{aligned}
$$

As compared to the first-order composite solution as given by the second equation of Eq. (52), besides the additional second-order term $q(h)$, we re-evaluate all the constants of integration to construct a modified second-order solution in the form

$$
\begin{aligned}
\cos \gamma_{m}^{c} & =\cos \gamma_{m c}+q_{m c} \\
& =\frac{C_{2}^{\prime}}{\sqrt{C_{1}^{\prime}(1+h)^{2}+2(1+h)}}+B \lambda \cos \sigma e^{-h / \varepsilon}+q_{m c}(h)
\end{aligned}
$$

such that

$$
B \lambda \cos \sigma e^{-h_{b}^{\prime} / \varepsilon}+\bar{C}_{2}^{\prime}+q_{m c}\left(h_{b}^{\prime}\right)=1
$$


where $\cos \gamma_{m c}$ is the modified first-order composite solution and $q_{m c}$ is the composite solution for $q$ but now denoting the difference between the $\cos \gamma_{m c}$ and the exact solution. The solution for $u$ is the modified first-order solution with new values for the constants and the bottom altitude.

$$
u_{m c}=\frac{2}{1+h}+\bar{C}_{1}^{\prime} e^{-\frac{\left(1+\lambda^{2}\right)}{\lambda E^{\prime} \cos \sigma} \cos ^{-1}\left(B \lambda \cos \sigma e^{-h / \varepsilon}+\bar{C}_{2}^{\prime}\right)}-\frac{2}{1+h_{b}^{\prime}}
$$

Equations (65) and (67) are now the improved compositc solutions for $u$ and $\gamma$ in three-dimensional skip trajectory. The constants of integration $C_{1}^{\prime}, C_{2}^{\prime}, \bar{C}_{1}^{\prime}, \bar{C}_{2}^{\prime}$ and the lowest altitude $h_{b}^{\prime}$ are evaluated from the equation (66), together with the first two of equations (52) with the accented constants and the two equations (65) and (67) satisfying the initial conditions $u_{m c}=u_{i}$ and $\gamma_{m}^{c}=\gamma_{i}$ at the initial altitude $h=h_{i}$.

For the second-order solution of $\psi$, by inspecting the third equation of the perturbation equations (55), we observe that the equation for the perturbation $y$ has the same difficulty in sensitivity as we encountered in solving the perturbation $z$. We then concentrate on solving the second-order solution for $\phi$ and $\theta$.

\section{Second-Order Solutions for $\phi$ and $\theta$}

The second-order solutions for $\phi$ and $\theta$ are obtained by integrating the perturbation equations for $f$ and $p$ in equations (55). The solutions in the outer region are trivially zero. For the inner region, by changing the independent variable to $\bar{\gamma}_{o}$ for the integration, we obtain

$$
\begin{aligned}
\bar{f}_{o}= & \left\{\tan \sigma\left[F_{1}\left(\bar{\gamma}_{o}\right)+\bar{C}_{2} F_{2}\left(\bar{\gamma}_{o}\right)\right]+\bar{C}_{3}\left[\bar{\gamma}_{o}+\bar{C}_{2} F_{3}\left(\bar{\gamma}_{o}\right)\right]+\cdots\right. \\
& \left.+\frac{\sin \psi_{o o}}{\tan \gamma_{o o}} \ln \left(\cos \bar{\gamma}_{o}-\bar{C}_{2}\right)\right\} \frac{1}{\left(1+h_{b}\right)}+\bar{C}_{9} \\
\bar{p}_{o}= & \left\{\bar{\gamma}_{o}+\bar{C}_{2} F_{3}\left(\bar{\gamma}_{o}\right)+\frac{\cos ^{2} \psi_{o o}}{C_{3} \tan \gamma_{o o}} \ln \left(\cos \bar{\gamma}_{o}-\bar{C}_{2}\right)\right. \\
& -\frac{1}{2 !}\left[2 \bar{C}_{3} \tan \sigma\left(F_{1}\left(\bar{\gamma}_{o}\right)+\bar{C}_{2} F_{2}\left(\bar{\gamma}_{o}\right)\right)+\cdots\right\} \frac{1}{\left(1+h_{b}\right)} \\
& +\bar{C}_{10}
\end{aligned}
$$

where $\bar{C}_{9}$ and $\bar{C}_{10}$ are the corresponding constants of integration. Notice that the prime for $\bar{C}_{2}$ and $h_{b}$, which represents the new constants of integration, is omitted for reason of simplicity. In the solutions (68) we have defined the following functions

$$
\begin{aligned}
& F_{1}\left(\bar{\gamma}_{o}\right) \equiv \int\left[\ln \left(1+\sin \bar{\gamma}_{o}\right)-\ln \left(\cos \bar{\gamma}_{o}\right)\right] d \bar{\gamma}_{o} \\
& =-\cos \bar{\gamma}_{o}+\frac{1}{9}\left(-3 \cos \bar{\gamma}_{o}+\cos ^{3} \bar{\gamma}_{o}\right)+\cdots \\
& F_{2}\left(\bar{\gamma}_{o}\right) \equiv \int \frac{\ln \left(1+\sin \bar{\gamma}_{o}\right)-\ln \left(\cos \bar{\gamma}_{o}\right)}{\cos \bar{\gamma}_{o}-\bar{C}_{2}} d \bar{\gamma}_{o} \\
& =-\ln \left(\cos \bar{\gamma}_{o}-\bar{C}_{2}\right)-\frac{1}{3}\left[-\frac{1}{2} \cos ^{2} \bar{\gamma}_{o}-\bar{C}_{2} \cos \bar{\gamma}_{o}\right. \\
& \left.+\mu \ln \left(\cos \bar{\gamma}_{o}-\bar{C}_{2}\right)\right]+\cdots \\
& F_{3}\left(\bar{\gamma}_{o}\right) \equiv \int \frac{1}{\cos \bar{\gamma}_{o}-\bar{C}_{2}} d \bar{\gamma}_{o} \\
& =\frac{-1}{\sqrt{\mu}}\left[\ln \left(\frac{\mp \sqrt{\mu} \sin \bar{\gamma}_{o}-\bar{C}_{2} \cos \bar{\gamma}_{o}+1}{\cos \bar{\gamma}_{o}-\bar{C}_{2}}\right)+\ln 2\right]
\end{aligned}
$$

and

$$
\mu \equiv 1-\bar{C}_{2}^{2}
$$

where we take minus sign for descending arc and plus sign for ascending arc in functions (69). Since the outer solutions for the perturbations $f$ and $p$ are zero, the composite solutions for $f$ and $p$ are identical to the inner solutions (68). Then, combining the fourth and fifth of the outer solutions (44) with the inner solutions (68), the second-order solutions for $\phi$ and $\theta$ are of the form

$$
\begin{aligned}
& \phi^{c}=\phi_{c}+f_{c}=\phi_{o}+\varepsilon \bar{f}_{o} \\
& \theta^{c}=\theta_{c}+p_{c}=\theta_{o}+\varepsilon \bar{p}_{o}
\end{aligned}
$$

The structure of the solutions is elegant in the sense that it reveals the physical phenomenon in terms of mathematic description. The first term in equations (71) represents the Keplerian motion without atmospheric influence. On the other hand, the second term is the small perturbations due to the effect of aerodynamic force in the inner region.

\section{Numerical Applications}

The purpose of this section is to discuss the relative merits and the ranges of application for the analytic solutions obtained by using the improved method of matched asymptotic expansions. As numerical examples, we consider the three-dimensional motion of a space vehicle entering the Earth's atmosphere. The vehicle has a maximum lift-to-drag ratio of $E^{*}=1$ with physical parameter $B=2.68$, starting with the initial entry speed $V_{i}=1.1 V_{c}$, i.e., $u_{i}=1.21$ and using $\lambda=1, \sigma=30^{\circ}$. Without loss of generality, we set other variables at the initial entry altitude $h_{i}=0.0157$ to be zero, that is $\left[\psi_{i}, \phi_{i}, \theta_{i}\right]=[0,0,0]$. Fig. 5 and 6 plot the speed 
ratio $V / \sqrt{g_{s} r_{s}}$ and the flight path angle $\gamma$ as functions of the altitude. The dashed lines indicate the numerical solutions while the solid lines represent the iMAE solutions. The accuracy of the solutions is cxcellent since, as shown in Table 1, we have obtained 6 significant digits at the lowest point. Table 1 presents the numerical results of the trajectory variables at the bottom and at exit. In each box the upper value is the pure numerical solution while the lower value is computed by our analytical solutions. From this table, we see that the relative error for the speed is about $0.2 \%$ at the bottom while it propagates to $0.7 \%$ at exit for the worst case of $-\gamma_{i}=4^{\circ}$.

Table 1 Comparison of the Trajectory Elements $(\sqrt{u}, \gamma)$ at Bottom and at Exit for Various Entry Angles

\begin{tabular}{|c|c|c|c|c|}
\hline$-\gamma_{i}$ & $4^{\circ}$ & $6^{\circ}$ & $8^{\circ}$ & $12^{\circ}$ \\
\hline$h_{b}$ & 0.0084587 & 0.0070730 & 0.0062788 & 0.0052702 \\
& 0.0084470 & 0.0070704 & 0.0062783 & 0.0052708 \\
\hline$u_{b}^{1 / 2}$ & 1.0516406 & 1.0009038 & 0.9570115 & 0.8788922 \\
& 1.0466288 & 0.9989403 & 0.9562586 & 0.8791299 \\
\hline \multirow{2}{*}{$u_{e}^{1 / 2}$} & 0.9901114 & 0.8918307 & 0.8117041 & 0.6781483 \\
& 0.9828935 & 0.8914917 & 0.8137913 & 0.6819361 \\
\hline \multirow{2}{*}{$-\gamma_{e}$} & 2.8186071 & 3.8039602 & 5.1206997 & 7.9369104 \\
& 2.7141180 & 3.8122696 & 5.1839038 & 8.0714158 \\
\hline
\end{tabular}

Since the $\mathrm{MAE}$ solutions are very accurate throughout the descending phase, the solutions are competent for the evaluation of the critical elements at the peak deceleration and the maximum heating, which always occur before the lowest point is reached. However, due to the basic condition of MAE technique, the solutions are less accurate for entry at small angles when the various forces involved are of the same order of magnitude throughout the trajectory. A completely different approach is suggested to analyze the case of entry with small angles 9 . Figs. 7 - 9 present the variations of the heading angle, latitude and longitude as functions of the altitude. It is seen that for the initial entry angle $-\gamma_{i}=4^{\circ}$, there is some discrepancy between the numerical solution and the MAE solution, as shown in Fig. 8. Even though the relative error for this case is large, the difference is only $0.069^{\circ}$ in absolute value for the latitude at exit. Table 2 provides the comparison of numerical results at exit for various entry angles.

Table 5.2 Comparison of the Trajectory Elements $(\psi, \phi, \theta)$ at Exit for Various Initial Entry Angles

\begin{tabular}{|c|c|c|c|c|}
\hline$-\gamma_{i}$ & $4^{\circ}$ & $6^{\circ}$ & $8^{\circ}$ & $12^{\circ}$ \\
\hline$\psi_{e}$ & 2.9344642 & 5.8764035 & 8.5237840 & 13.559225 \\
& 3.1321319 & 5.8789130 & 8.4401482 & 13.382860 \\
\hline$\phi_{e}$ & 0.5140644 & 0.7370882 & 0.8241616 & 0.9069345 \\
& 0.5829793 & 0.7557625 & 0.8289606 & 0.9037549 \\
\hline$\theta_{e}$ & 18.603317 & 13.167788 & 10.178101 & 7.0592236 \\
& 19.332678 & 13.354608 & 10.307469 & 7.2076078 \\
\hline
\end{tabular}

To study the effect of the bank angle on the threedimensional skip trajectory, we consider the atmospheric entry with the same initial speed and altitude at the initial entry angle $-\gamma_{i}=6^{\circ}$ for three values of bank angles $\sigma=$ $30^{\circ}, 45^{\circ}$, and $60^{\circ}$. The variations of the trajectory variables as functions of the altitude for these cases are plotted in Figs. 10 - 12. Again the dashed lincs represent the numerical solutions and the solid lines represent the iMAE solutions. It is noted that the space vehicle fails to skip out with the bank angle $60^{\circ}$ and the relative error becomes larger when the bank angle is larger. Nevertheless, in Figs. 11 and 12, the iMAE solutions for latitude and longitude remain in good agreement with the numerical solutions for the three bank angles.

\section{Conclusions}

In this paper, an improved method of matched asymptotic expansions has been proposed for deriving the second-order solutions of non-linear differential equations which govern dynamic systems with controlling forces varying widely between the end-points. The improved solutions obtained by the proposed techniquc are expressed in two parts: the first-order composite solutions and the composite solutions of the perturbations which are the differences between the first-order composite solutions and the exact solutions. The first-order composite solutions and the composite solutions of the perturbations consist of outer solutions, inner solutions, and the common limits of the outer and inner solutions. To investigate the applicability and the efficiency of the proposed technique, we first applied it to the rectilinear restricted three-body problem. Next, we applied it to analyze the threedimensional atmospheric re-entry problem. In the process, for the matching we extend the end-point in the outer region to validate the assumptions of outer expansions with one dominant force, while we create the boundary condition for the inner limit to strictly enforce the strong aerodynamic force assumption of inner expansions.

The improved MAE solutions are very accurate when compared with the pure numerical solution of the equations of motion. For skip trajectory with an error in the sixth or seventh digit for the altitude, it is not necessary to go to further higher-order terms to improve on the final solutions. Along with the solutions for speed and flight path angle, the analytic solutions for heading change, latitude and longitude are obtained and tested in a wide range of entry conditions. The accuracy of the analytic solutions was satisfactorily assessed cvaluated at the lowest altitude and at exit. The numerical results from the analytic solutions show an excellent agreement with the pure numerical solutions. Since the solutions are in explicit form and uniformly valid in both the Keplerian and the atmospheric regions, the trajectory elements at exit can be expressed explicitly.

In conclusion, an improved method of matched asymptotic expansions has been developed to analyze the three-dimensional motion of the atmospheric skip 
trajectory. The second-order solutions obtained by using the improved method apply to all phases of flight from the vacuum through the atmosphere to either skip out or effective entry with a high degree of accuracy. The numerical results clearly demonstrate the applicability and flexibility of the improved technique.

\section{References}

[1] Prandtl, L., "Motion of Fluids with Very Little Viscosity," NACA Translation No. 452, 1928.

[2] Bretherton, F. P., "Slow Viscous Motion Round a Cylinder in a Simple Shear," J. Fluid Mech., Vol. 12, 1962, pp. 591-613.

[3] Lagerstrom, P. A., and Kevorkian, J., "Earth-toMoon Trajectories in the Restricted Three-Body Problem," J. Mécanique, Vol. 2, 1963, pp. 189-218.

[4] Breakwell, J. V., and Perko, L. M., "Matched Asymptotic Expansions, Patched Conics, and the Computation of Interplanetary Trajectories," in Progress in Astronautics and Aeronautics, Vol. 17, R. L. Duncombe and V. G. Szebehely Eds., Academic Press, New York, 1966, pp. 159-182.

[5] Shi, Y. Y., Pottsepp, L., and Eckstein, M. C., "A Matched Asymptotic Solution for Skipping Entry into Planetary Atmosphere," AIAA Journal, Vol. 9, No. 4 1971, pp. 736-738.

[6] Shi, Y. Y., "Matched Asymptotic Solution for Optimum Lift Controlled Atmospheric Entry," AIAA Journal, Vol. 9, No. 11, 1971, pp. 2229-2238.

[7] Busemann, A., Vinh, N. X., and Culp, R. D., "Solution of the Exact Equations for ThreeDimensional Atmospheric Entry Using Directly Matched Asymptotic Expansions," NASA Report CR-2643, 1976.

[8] Nayfeh, A. H., Perturbation Methods, John Wiley \& Sons, Inc., NY, 1973.

[9] Longuski, J. M., and Vinh, N. X., "Analytic Theory of Orbit Contraction and Ballistic Entry into Planetary Atmospheres," JPL Publication 80-58, September 1980.

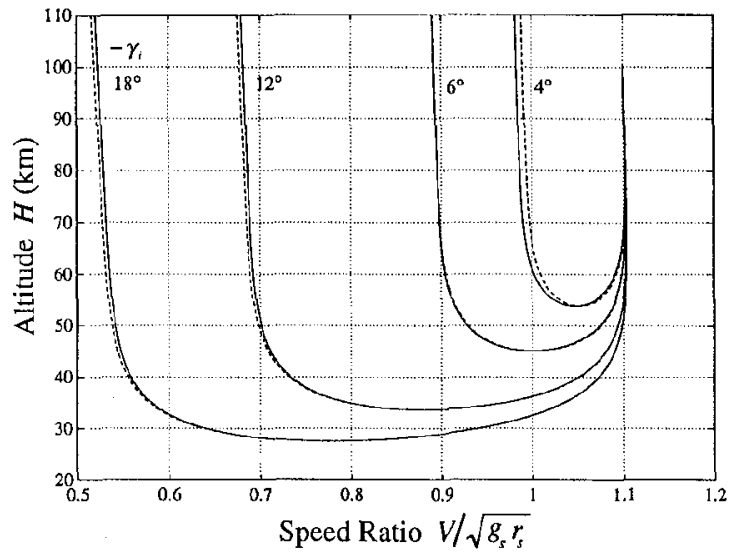

Fig. 5 Variation of speed as function of the altitude for various entry angles in three-dimensional skip trajectory

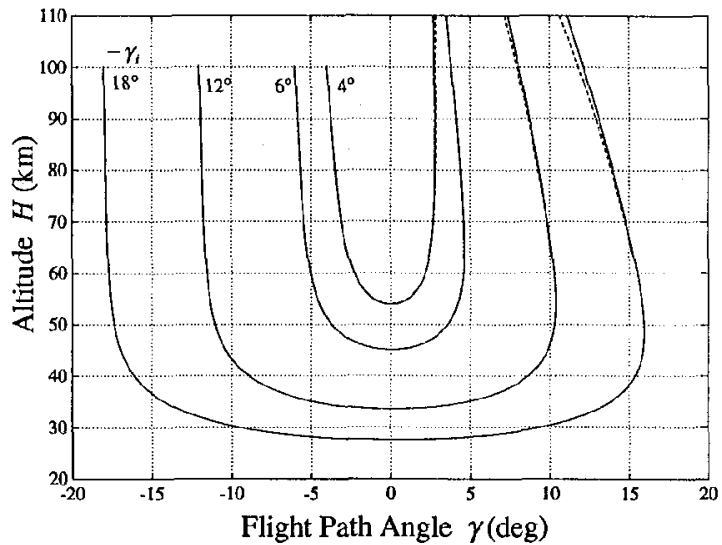

Fig. 6 Variation of flight path angle as function of the altitude for various entry angles in threcdimensional skip trajectory

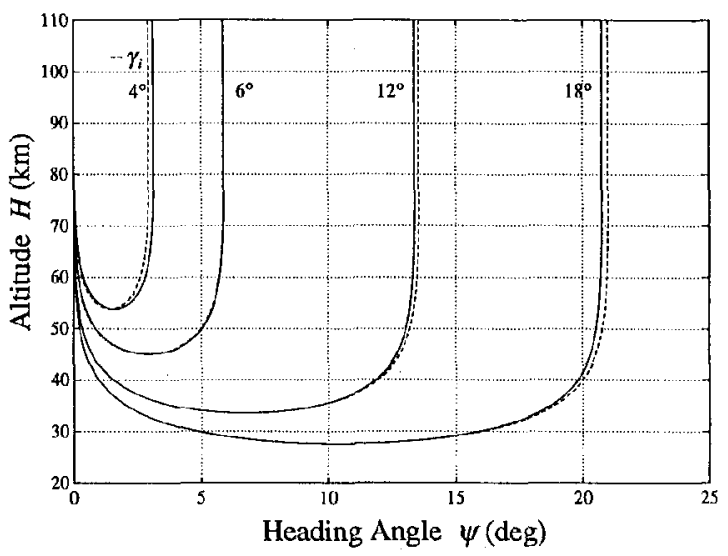

Fig. 7 Variation of heading angle as function of the altitude for various entry angles in threedimensional skip trajectory

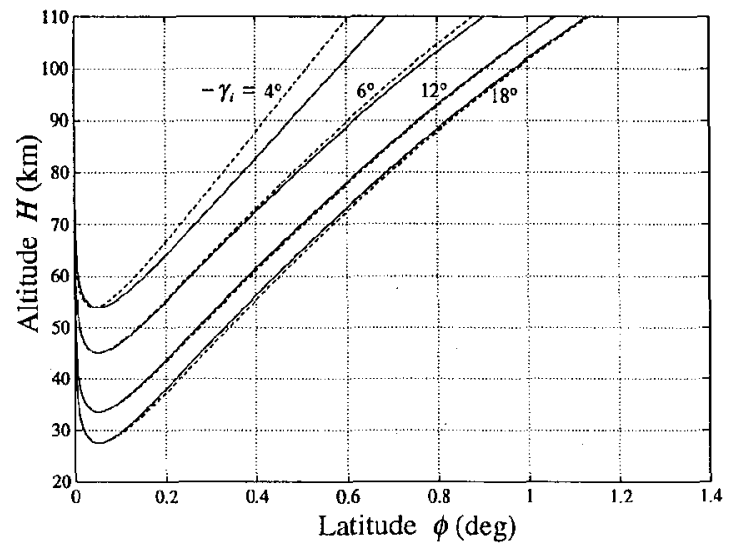

Fig. 8 Variation of latitude as function of the altitude for various entry angles in three-dimensional skip trajectory 


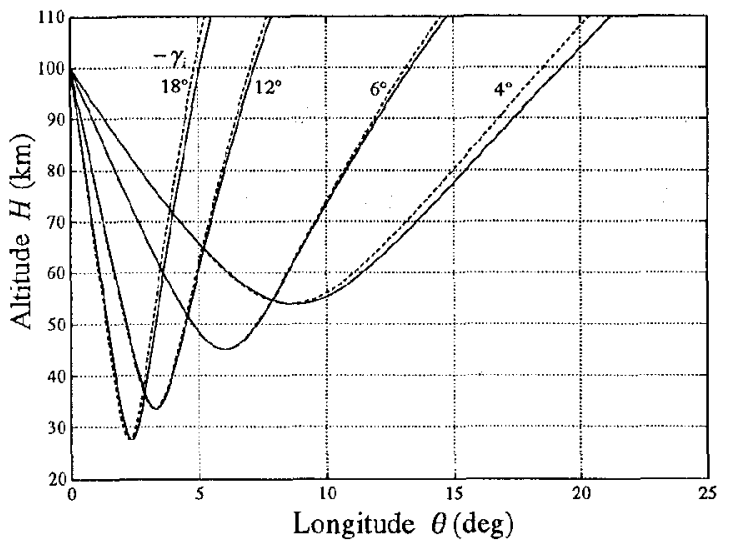

Fig. 9 Variation of longitude as function of the altitude for various entry angles in three-dimensional skip trajectory

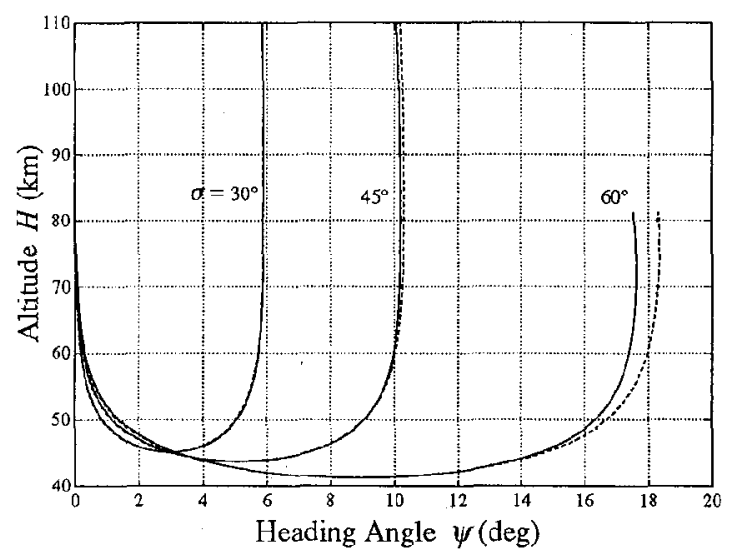

Fig. 10 Variation of heading angle as function of the altitude for various bank angles in threedimensional skip trajectory

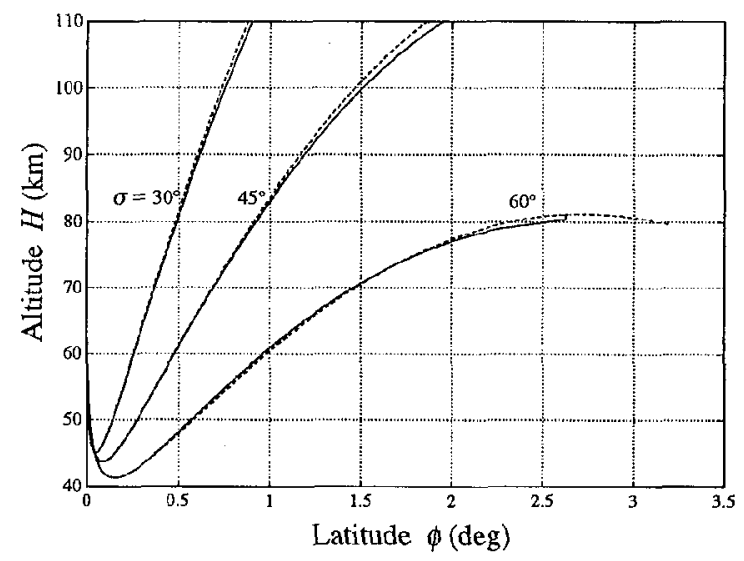

Fig. 11 Variation of latitude as function of the altitude for various bank angles in three-dimensional skip trajectory

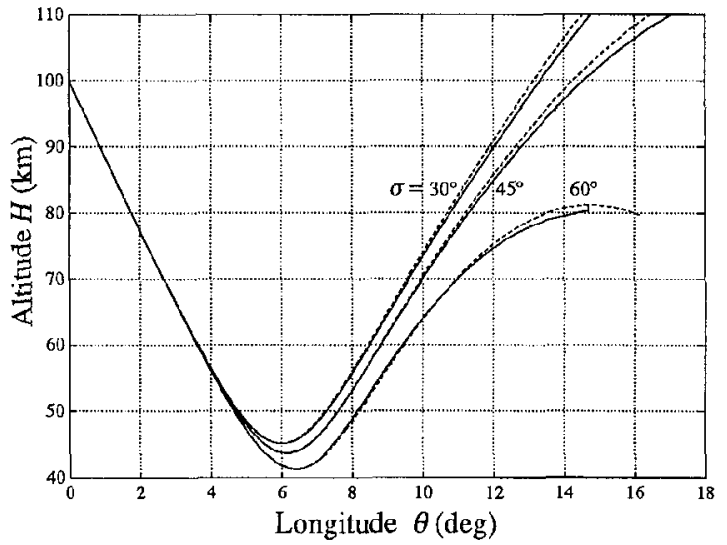

Fig. 12 Variation of longitude as function of the altitude for various bank angles in three-dimensional skip trajectory 